\title{
Selective expression of S100A7 in lung squamous cell carcinomas and large cell carcinomas but not in adenocarcinomas and small cell carcinomas
}

\author{
H Zhang, 0 Zhao, ${ }^{1}$ Y Chen, ${ }^{1}$ Y Wang, ${ }^{2}$ S Gao, ${ }^{3}$ Y Mao, ${ }^{1}$ M Li, ${ }^{1}$ A Peng, ${ }^{1}$ D He, ${ }^{1}$ X Xiao'
}

${ }^{1}$ Key Laboratory of Cell

Proliferation and Regulation

Biology, Ministry of Education,

Beijing Normal University, and

Universities of Confederated

Institute for Proteomics, Beijing,

China; ${ }^{2}$ Department of

Pathology, First Hospital of Jilin

University, Changchun, China;

${ }^{3}$ Department of Chest Surgery,

Cancer Hospital, Peking Union

Medical College and Chinese

Academy of Medical Science,

Beijing, China

\section{Correspondence to:}

Dr X Xiao, Dacheng He, Key

Laboratory of Cell Proliferation

and Regulation Biology, Ministry

of Education, Beijing Normal

University, 19th Xin jie kou wai

da jie, Beijing 100875, China;

xyxiao@bnu.edu.cn

Received 8 July 2007

Accepted 4 October 2007

\begin{abstract}
Background: S100A7 is a secreted protein and its overexpression has been previously associated with carcinogenesis of certain cancers. This study was undertaken to investigate the possibility that overexpression of S100A7 protein might be detected in the sera of patients with lung cancer.
\end{abstract}

Methods: RNA and protein levels of S100A7 were examined in 60 pairs of frozen lung cancer tissues by RTPCR and western blot. The specific expression of this protein and its cellular distribution were investigated in 145 paraffin embedded lung cancer samples, six benign lung disease and 21 normal lung tissues by immunohistochemistry. The S100A7 protein level was further analysed in serum from 112 patients with lung cancer, 20 with benign lung diseases and 31 healthy individuals by ELISA.

Results: Specific expression of both S100A7 mRNA and protein was found in squamous cell carcinomas, adenosquamous carcinomas and large cell lung carcinomas, whereas neither was detected in adenocarcinomas or paired non-cancerous lung tissues. Further immunohistochemical analysis identified positive staining of S100A7 only in squamous cell carcinomas and large cell lung carcinomas, but not in other subtypes of lung cancer and normal lung tissues. Weak expression was also found in the inflammatory cells of benign lung diseases. Our most important finding is that elevated S100A7 protein could be detected in the sera of patients with squamous cell carcinomas.

Conclusion: S100A7 was only expressed in squamous cell carcinomas and large cell lung carcinomas and an increase in the level of S100A7 protein in serum may serve as a potential marker for lung cancer diagnosis.

Lung cancer is a leading cause of malignancy related deaths worldwide, and the 5 year patient survival rates remain $14 \%$, despite diagnostic imaging and therapy improvements over the past decades. ${ }^{12}$ Although established biomarkers such as p53, NES, CEA, Cyfra21-1 and CA19-9 are commonly used as lung cancer biomarkers, ${ }^{34}$ several potential biomarkers, such as SAA and TTR, have been investigated recently in lung cancer. ${ }^{5}{ }^{6}$ However, very few have been accepted for clinical diagnosis, progression and/or prognosis, either because of the lack of specificity or because of conflicting reports. Therefore, $65 \%$ of patients with lung cancer with advanced diseases at the time of diagnosis lose the best therapeutic opportunity for surgical resection because of lack of good biomarkers.? More effective management of lung cancer is required to identify the specific markers at a stage early enough to be curable by surgery.
S100A7 (psoriasin) belongs to the S100 multigenic family of calcium modulated proteins of the $\mathrm{EF}$ hand type and is relatively unique in structure. The locus encoding the S100A7 gene was duplicated in evolution and is composed of three distinct genomic regions. Regions 1 and 3 are in opposite orientation to each other, each carries two S100A7-like genes separated by an $11 \mathrm{~kb}$ intergenic region (region 2). Region 2 also contains an S100A7-like gene. ${ }^{8}$ S100A7 was originally identified as a protein whose expression was increased in psoriatic keratinocytes. ${ }^{9}$ Later studies have shown that S100A7 could play an important role in antibacterial action $^{10}$ and its overexpression is prominently associated with early stages of various carcinomas, including squamous carcinomas in skin and bladder, and ductal in situ carcinomas and adenocarcinomas of the breast. ${ }^{11-16}$ Expression of S100A7 is often diminished in carcinomas during their progression to invasive carcinoma. However, persistent S100A7 expression can occur in subsets of invasive breast carcinomas associated with the worse clinical outcome. ${ }^{17}$ Although S100A7 is present in the cytoplasm and nucleus of abnormally differentiated keratinocytes and breast carcinoma cells, ${ }^{15}$ secreted S100A7 was found in psoriatic skin lesions and in amniotic fluid and urine of patients with squamous cell carcinoma. ${ }^{18-20}$ Because of these observations, we wondered whether S100A7 could be a potential serum marker for lung cancer. To test this possibility, we first analysed various tissues to determine if there is differential expression of S100A7 between primary lung cancer tissues and matched non-cancerous lung tissues, or between benign lung diseases and their normal counterparts. We then examined whether the S100A7 protein can be detected in the serum of patients with lung cancer and control groups and whether its detection is limited to lung cancer or subtypes of lung cancer. Finally, we tested whether the association of S100A7 with lung cancer is statistically significant and whether it can serve as a serum marker for lung cancer.

\section{MATERIALS AND METHODS}

\section{Tissue and serum specimens}

Sixty frozen lung cancer tissues and matched noncancerous lung tissues (average $5 \mathrm{~cm}$ ) from the same patients, one skin biopsy specimen from a patient with chronic plaque-type psoriasis, 112 serum samples from patients with lung cancer and 20 serum samples from patients with benign lung disease were obtained from the Cancer Hospital, Peking Union Medical College and Chinese 
Academy of Medical Science and General Hospital of Beijing Unit, PLA. The frozen tissues obtained during surgery were snap frozen and stored in liquid nitrogen. The lung cancer serum samples were from 86 males and 26 females, aged $21-82$ years, and the benign lung disease serum samples were from 15 males and five females, aged 25-78 years. Among 145 formalin fixed and paraffin embedded lung cancer tissues, 65 were provided by the First Hospital of Jilin University, two cases (adenosquamous cell carcinomas) were collected from General Hospital of Beijing Unit, PLA and 78 were obtained from two microarrays (lung cancer tissues No CC04-11-005 and normal lung tissues No NC04-01001) purchased from Cybrdi Co. Ltd (Xian, China). No CC04-11005 tissue array comprised 28 squamous cell carcinomas, 17 adenocarcinomas, 11 small cell carcinomas, three large cell carcinomas, three alveolar carcinomas, five adenosquamous carcinomas, four mucoid adenocarcinomas, three papilla adenocarinomas and one carcinoid tumour of lung, two pneumonia and three normal lung tissues. No NC04-01-001 array comprised two adenocarcinomas, one squamous cell carcinoma, four benign lung diseases and 18 normal lung tissues. Three punches of each sample were arrayed on the latter slide. All patients with lung cancer had a pathological diagnosis and none had received prior therapy. Lung cancer staging was based on the tumour-node-metastasis and staging classification of $1989^{21}$ and was comparable with the new WHO criteria classification of 1999 . Thirty-one normal control serum samples from 23 males and eight females, aged 23-75 years, were obtained from a general health examination from the staff of the General Administration of Sport. Processing, collection and storage protocols for serum samples for normal individuals were the same as those for the patients. All samples were collected between December 2005 and July 2006, and written informed consents was obtained from each donor. The study was approved by the Medical Ethics and Human Clinical Trial Committee at First Hospital of Jilin University and Cancer Hospital, Peking Union Medical College and Chinese Academy of Medical Science, as well as General Hospital of Beijing Unit, PLA.

\section{RNA isolation and reverse transcription (RT)}

Total RNA was isolated from tissues using Trizol reagent (Invitrogen, Carlsbad, California, USA), and $2 \mu \mathrm{g}$ of RNA were reverse transcribed into single strand cDNA using molony murine leukaemia virus reverse transcriptase (Promega, Madison, Wisconsin, USA) and oligo (dT) 15 (Promega) as a primer. Normal tissue cDNA panels (multiple tissue cDNA panels (MTC) I and II) composed of brain, colon, heart, kidney, leucocytes, liver, lung, ovary, pancreas, placenta, prostate, skeletal muscle, small intestine, spleen, thymus and testis were purchased from BD Biosciences Clontech (Palo Alto, California, USA).

\section{Semiquantitative PCR}

Semiquantitative PCR reactions were carried out with S100A7 gene specific primers (5'-AAC TTC CTT AGT GCC TGT G-'3 and 5'-TGG TAG TCT GTG GCT ATG TC-'3) or with $\beta$-actin (ACTB) specific primers (5'-AAA TCT GGC ACC ACA CCT TC-'3 and 5'-GGG GTG TTG AAG GTC TCA AA-'3) as an internal control. All PCR reactions were programmed for an initial denaturation at $94^{\circ} \mathrm{C}$ for $5 \mathrm{~min}$ followed by 23 (for ACTB) or 27 cycles (for S100A7) at $94^{\circ} \mathrm{C}$ for $30 \mathrm{~s}, 55^{\circ} \mathrm{C}$ for $30 \mathrm{~s}$ and $72^{\circ} \mathrm{C}$ for $60 \mathrm{~s}$ on an ABI GeneAmp PCR System 9700 (Applied Biosystems, Foster City, California, USA). The PCR products were analysed by electrophoresis in 1\% agarose gel. The electrophoresis images were scanned with Flour-s
Multimager (Bio-Rad, California, USA), and the original intensities of specific bands were quantified by Multi-Analyst software (Bio-Rad). The amount of S100A7 expression was normalised to the ACTB endogenous reference standard and was presented as the ratio of S100A7:ACTB. The identity of the DNA product was confirmed by nucleotide sequencing. PCR experiments were repeated in triplicate on the samples with similar results.

\section{Western blot}

Tissue samples of homogenates of the lung cancer or matched non-cancerous lung were treated with detergent solution (62.5 mM EDTA, Tris ( $\mathrm{pH} 8.0$ ), 0.4\% deoxycholic acid and 1\% NP40). Equal quantities of protein were separated by $12 \%$ sodium dodecyl sulphate-polyacrylamide gel electrophoresis and transferred onto nitrocellulose membrane (Amersham Biosciences, Piscataway, New Jersey, USA) using a Bio-Rad wet transfer unit. After blocking with $5 \%(\mathrm{w} / \mathrm{v})$ non-fat dried milk in TBST solution (25 mM Tris, pH 7.5, $150 \mathrm{mM} \mathrm{NaCl}$, $0.05 \%(\mathrm{v} / \mathrm{v})$ Tween-20) for $1 \mathrm{~h}$ at room temperature, the membranes were incubated with S100A7 antibody (1:1000 dilution) for $1 \mathrm{~h}$ at room temperature, followed by alkaline phosphatase conjugated second antibody for $1 \mathrm{~h}$ at room temperature. Target proteins were detected by 5-bromo-4chloro-3-indoyl phosphate and nitroblue tetrazolium. $\beta$-Actin was used to normalise the loaded proteins. Monoclonal antibody against S100A7 was purchased from Imgenex (San Diego, California, USA, USA, catalogue No IMG-409A) and this antibody was developed against recombinant psoriasin/HD-5 protein).

\section{Immunohistochemistry}

Immunohistochemical staining was carried out on $10 \%$ formalin fixed, paraffin embedded tissue sections cut to $5 \mu \mathrm{m}$ thickness. All sections were dried at $60^{\circ} \mathrm{C}$ for $30 \mathrm{~min}$, treated with xylenes and then dehydrated in alcohol. Endogenous peroxidase was blocked with hydrogen peroxide (3\%) for $10 \mathrm{~min}$ to remove endogenous peroxidases, and non-specific binding was blocked with normal mouse serum. Microwave treatment of the slides was performed for $15 \mathrm{~min}$ in $0.01 \mathrm{mM}$ citrate buffer ( $\mathrm{pH} 6.0$ ). After incubating with blocking serum, the S100A7 antibody (1:200 dilution in phosphate buffered saline) was applied for $1 \mathrm{~h}$ at $37^{\circ} \mathrm{C}$ followed by washing and subsequent incubation with the secondary antibody, peroxidase conjugated affinity purified rabbit anti-mouse (1:200 dilution) for $1 \mathrm{~h}$ at $37^{\circ} \mathrm{C}$. Detection was performed with $3,3^{\prime}$-diaminobenzidine tetrahydrochloride peroxidase substrate and counterstaining with haematoxylin, followed by dehydration, clearing and mounting. A negative control (normal mouse serum only/no primary antibody) was performed in parallel. All immunostained slides for S100A7 were evaluated independently by two pathologists. Staining in cytoplasmic, nuclear and cytoplasmic membrane was considered positive. Cancer specimens containing $>5 \%$ cells for S100A7 staining was regarded as positive.

\section{ELISA}

Serum S100A7 level was examined by ELISA with commercial monoclonal antibody against S100A7. Individual serum samples were diluted 1:10 with coating buffer $(0.05 \mathrm{M}$ carbonate buffer, $\mathrm{pH}$ 9.6) and then $100 \mu \mathrm{l}$ of diluted serum were added to each well in a 96 well plate. After leaving overnight at $4^{\circ} \mathrm{C}$, the wells were washed three times with washing buffer $(1 \times$ phosphate buffered saline, $0.05 \%$ Tween $20, \mathrm{pH} 7.4$ ) and blocked with $3 \%$ 
Figure 1 Analysis of S100A7 transcript levels in 16 normal human tissues, using lesional psoriatic skin as a positive control. (A) Conventional RT-PCR analysis for S100A7 transcript. The housekeeping gene $\beta$-actin was used as a control. (B) The results are presented as the ratio of S100A7 gene expression to $\beta$-actin gene expression. Values are mean (SD).
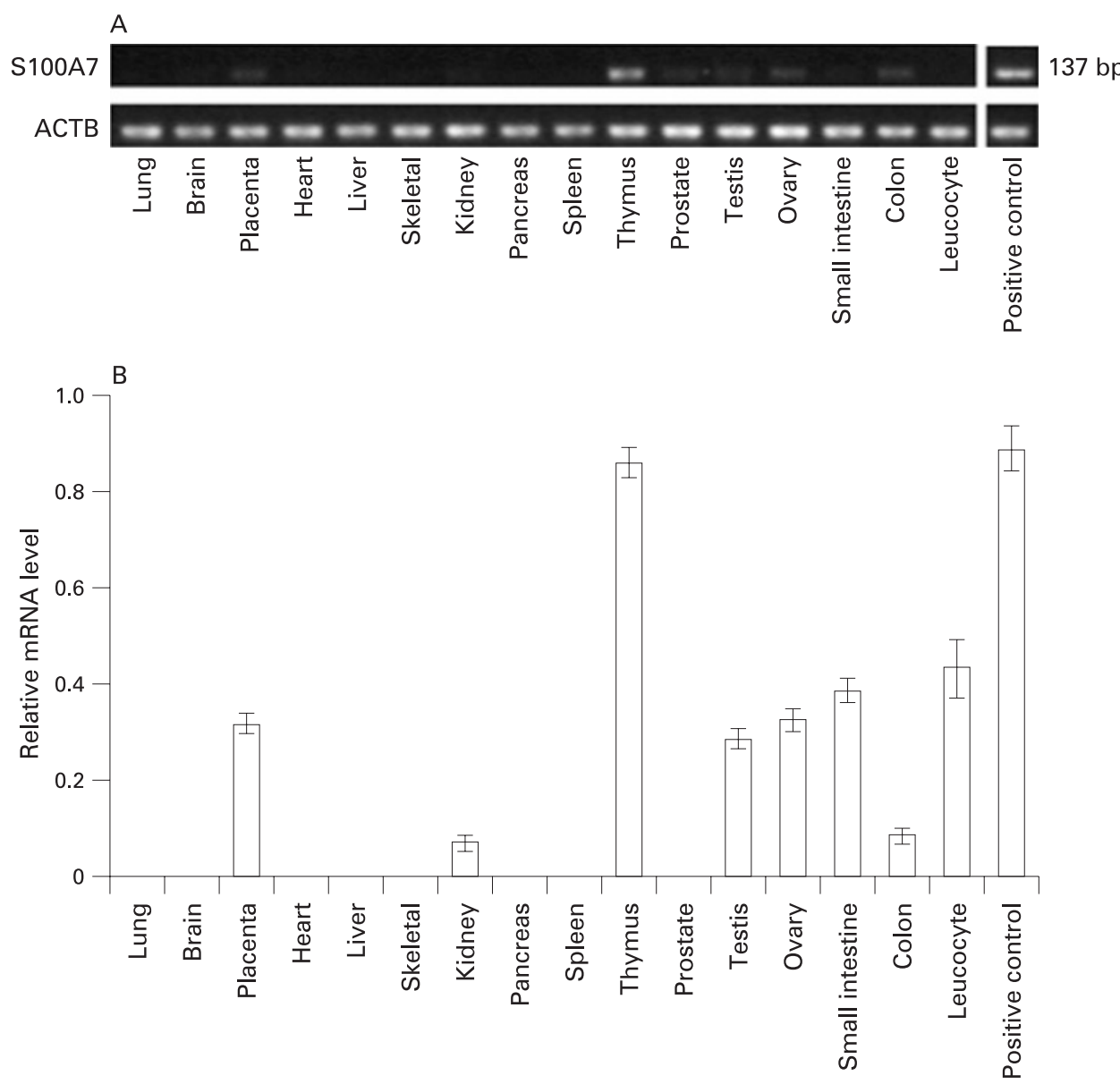

bovine serum albumin. Then, $100 \mu \mathrm{l}$ of S100A7 antibody (1:5000 dilution) were added and incubated for $1.5 \mathrm{~h}$ at $37^{\circ} \mathrm{C}$. After three washes, to each well was added $100 \mu$ l of secondary antibody (1:3000 diluted goat antimouse IgG-HRP antibody; Golden Bridge Co., China) and incubated for an additional $1.5 \mathrm{~h}$ at $37^{\circ} \mathrm{C}$. After washing, the reaction was developed with OPD and terminated by $3 \mathrm{M} \mathrm{H}_{2} \mathrm{SO}_{4}$. Absorbance was measured on a microplate reader (Bio-Rad model 550; Bio-Rad) at $490 \mathrm{~nm}$. The primary antibody depleted was used as a negative control. All analyses were assayed in duplicate.

\section{Statistical analysis}

The association between S100A7 expression and categorical groups was analysed by the $\chi^{2}$ test or Fisher's exact test, as appropriate. The Pearson correlation coefficient ( $r$ ) and associated probability $(\mathrm{p})$ were calculated to analyse the correlation between mRNA and protein levels of S100A7. A non-parametric test (Mann-Whitney $U$ test) was used to compare the ELISA results from different groups. Two tailed $p$ values of 0.05 or less were considered significant. Receiver operating characteristic analysis was used to detect the optimal cut-off points (ie, those with the highest total accuracy) for separating squamous cell carcinomas from other tested groups.

\section{RESULTS}

\section{S100A7 mRNA is not detectable in normal lung tissue}

S100A7 has been reported to be expressed in psoriatic keratinocytes and some cancer cells. S100A7 expressions in human multiple tissue cDNA panels (Clontech), including 16 normal human tissues, were first examined to determine its distribution and transcriptional levels. Expression levels of S100A7 varied significantly among 16 normal human tissues. The highest levels of S100A7 expression were found in the placenta, thymus, ovary and colon, while very low levels were detected in the prostate and testis. However, extremely weak or undetectable levels of S100A7 were found in the lung, brain, heart, kidney, leucocytes, liver, pancreas, skeletal muscle, small intestine and spleen (fig 1). These results demonstrate that S100A7 expression is not detectable in normal lung tissues.

\section{Expression of S100A7 in lung cancer tissues}

To determine if S100A7 could be a biomarker for lung cancer, we first confirmed whether S100A7 was differentially expressed in lung cancer and normal lung tissues. We performed semiquantitative PCR to analyse mRNA expression of S100A7 in 60 paired primary lung cancer tissues. To make the control as precise as possible, the corresponding normal bronchial epithelial cells were obtained by LCM (Palm MicroLaser System, Germany) from the matched non-cancerous lung tissue blocks. In difficult cases in which we could not obtain microdissected epithelial cells, the whole lung tissue blocks were used. We found that S100A7 was detected in squamous cell carcinomas, adenosquamous carcinomas and large cell lung carcinomas, but not in any of adenocarcinomas, normal bronchial epithelial cell samples or matched non-cancerous lung tissue blocks. To confirm whether S100A7 mRNA expression paralleled the protein level, S100A7 protein was also analysed in the same sets of tissue samples by western blot (table 1). Our data demonstrated that S100A7 gene expression correlated well with 
Table 1 S100A7 expression in human lung cancer and matched noncancerous tissue specimens

\begin{tabular}{llcc}
\hline Group & $\begin{array}{l}\text { No } \\
\text { tested }\end{array}$ & $\begin{array}{l}\text { mRNA expression } \\
\text { (No positive/No } \\
\text { tested) }\end{array}$ & $\begin{array}{l}\text { Protein expression } \\
\text { (No positive/No } \\
\text { tested) }\end{array}$ \\
\hline Non-cancerous lung tissue & 60 & $0 / 60$ & $0 / 60$ \\
Squamous cell carcinomas & 35 & $21 / 35$ & $18 / 35$ \\
I/II & 18 & $11 / 18$ & $9 / 18$ \\
III/IV & 17 & $10 / 17$ & $9 / 17$ \\
Adenocarcinomas & 18 & $0 / 18$ & $0 / 18$ \\
I/II & 10 & $0 / 10$ & $0 / 10$ \\
III/IV & 8 & $0 / 8$ & $0 / 8$ \\
Adenosquamous carcinomas & 5 & $2 / 5$ & $2 / 5$ \\
I/II & 3 & $1 / 3$ & $1 / 3$ \\
III/IV & 2 & $1 / 2$ & $1 / 2$ \\
Large cell lung carcinomas & 2 & $1 / 2$ & $1 / 2$ \\
I/II & 1 & $0 / 1$ & $0 / 1$ \\
III/IV & 1 & $0 / 1$ & $0 / 1$ \\
\hline
\end{tabular}

protein levels $(r=0.91 ; p=0.0015)$. However, there was no correlation between S100A7 expression and sex, age, clinical stages or nodular status. With regard to histology, squamous cell carcinomas showed higher S100A7 expression than other types of lung cancer $(p<0.001)$. Some representative results are shown in fig 2. For three squamous cell carcinomas with nondetectable S100A7, it is possible that S100A7 protein is expressed at extremely low levels, undetectable using our method. Nevertheless, our findings raise the possibility that S100A7 is expressed selectively in subtypes of lung cancer, independent of the clinical stage of the disease.

\section{Specific expression of S100A7 in squamous carcinomas and large cell carcinomas}

To confirm whether there is differential expression of S100A7 in subtypes of lung carcinomas and to determine its subcellular localisation, immunohistochemistry was initially performed in 67 formalin fixed, paraffin embedded lung cancer tissues which were obtained from two separate cohorts. When tissue samples were examined by histology and pathology, S100A7 immunoreactivity was scored from moderate to strong in squamous cell carcinomas, adenosquamous carcinomas and large cell carcinomas, respectively. However, the intensity of the immunoreactivity was independent of sex, age, clinical stages and nodular status. No immunoreactivity was observed in adenocarcinomas. This observation, together with our desire to see whether S100A7 was expressed in non-malignant lung diseases, prompted us to enlarge our screens to more and various types of lung cancer tissues. Among 78 cases of lung cancer tissues, six cases of benign lung disease tissues and 21 cases of normal lung tissues on two microarrays, we found that, as expected, some squamous cell carcinomas, large cell carcinomas and adenosquamous carcinomas were positive for immunoreactivity for S100A7. Taken together, among 145 cases of paraffin embedded lung cancer tissues, S100A7 positive specimens were observed in $53 \%$ of squamous cell carcinomas, $33 \%$ of adenosquamous carcinomas and $20 \%$ of large cell carcinomas. No immunoreactivity was detected in other subtypes of lung cancer or in normal lung tissues (table 2). Moreover, the positive staining of S100A7 in adenosquamous carcinoma tissues was only present in the squamous cell carcinoma area and weak immunoreactivity was also observed in some inflammatory cells of benign lung diseases, especially the alveolar macrophages. Representative immunoreactivity results are shown in fig 3. In addition, examination of the subcellular localisation of S100A7 protein in squamous cell carcinomas and large cell carcinomas revealed that it was detectable in nuclei and cytoplasm, occasionally in the plasma membrane but predominately localised in well differentiated squamous cell carcinomas. No correlation of S100A7 expression with sex, age, clinical stage or nodular status was observed. Histologically, squamous cell carcinoma tissues showed higher S100A7 expression levels than that of other types of lung cancer tissues $(p<0.001)$. Collectively, these findings suggest that S100A7 is highly and differentially expressed in squamous cell carcinomas and large cell carcinomas and high levels of its expression may serve as a useful marker for these types of lung carcinomas.

\section{The level of S100A7 protein increases in sera from squamous carcinoma patients}

Based on the above results and the observation that S100A7 was found in amniotic fluid and urine of patients with squamous cell carcinomas, ${ }^{19}{ }^{20}$ we next evaluated the potential diagnostic significance of S100A7 protein as a serum marker. For this purpose, an ELISA developed specifically for S100A7 detection was selected to analyse S100A7 serum levels in various groups of patients with lung cancer, individuals with benign lung diseases and healthy controls. It was found that the level of S100A7 in lung squamous cell carcinoma patients was significantly increased compared with other cell types of lung cancer and benign lung diseases $(p<0.001)$, the latter exhibiting a value that was similar to that of healthy individuals (table 3). S100A7 levels in differential grades and clinical stages of patients with lung cancer were elevated, with no significant differences. These data indicate that elevated S100A7 serum levels are specifically associated with lung cancer but not with benign lung diseases.

A
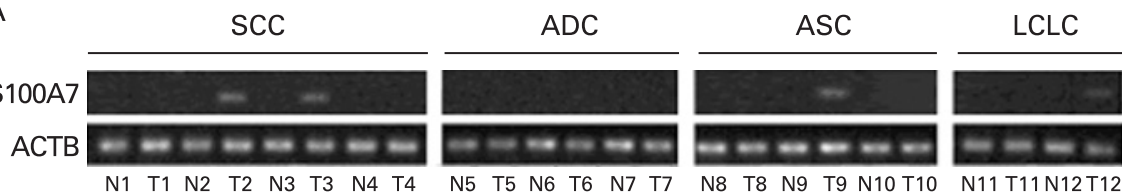

$137 \mathrm{bp}$

$\begin{array}{llllllll}\mathrm{N} 1 & \mathrm{~T} 1 & \mathrm{~N} 2 & \mathrm{~T} 2 & \mathrm{~N} 3 & \mathrm{~T} 3 & \mathrm{~N} 4 & \mathrm{~T} 4\end{array}$

N8 T8 N9 T9 N10 T10

N11 T11 N12T12
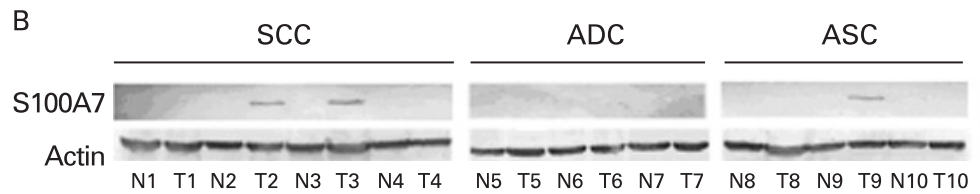

LCLC

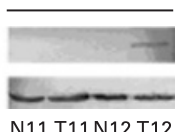

$11.7 \mathrm{kDa}$

Figure 2 (A) Conventional RT-PCR analysis for expression of S100A7 in subtypes of lung cancer tissues (T), including squamous cell carcinoma (SCC) adenocarcinoma (ADC), adenosquamous carcinomas (ASC) and large cell lung carcinoma (LCLC), and the matched adjacent non-cancerous tissues (N). (B) Western blot analysis of S100A7 protein in the same sets of tissues samples which were arranged in the same order as shown in (A). 
Table 2 S100A7 immunostaining in lung tissue specimens

\begin{tabular}{lcccc}
\hline \multirow{2}{*}{ Group } & \multirow{2}{*}{$\begin{array}{c}\text { Total No positive/ } \\
\text { No tested }\end{array}$} & \multicolumn{3}{l}{ S100A7 expression (No positive/No tested) } \\
\cline { 4 - 5 } & $3 / 62$ & $21 / 38$ & III/IV & Unstaged \\
\hline Squamous cell carcinomas & $33 / 62$ & $0 / 26$ & $12 / 23$ & $0 / 1$ \\
Adenocarcinomas & $0 / 45$ & $1 / 3$ & $1 / 2$ & - \\
Adenosquamous carcinomas & $3 / 9$ & $0 / 2$ & $1 / 2$ & $1 / 4$ \\
Large cell carcinomas & $1 / 5$ & - & $0 / 11$ & - \\
Small cell carcinomas & $0 / 11$ & $0 / 8$ & - & $0 / 5$ \\
Other lung cancers* & $0 / 13$ & & & \\
Normal lung & $0 / 21$ & & & \\
Benign lung diseases & $0 / 6$ & & & \\
\hline
\end{tabular}

*0ther lung cancers include alveolar carcinomas, mucoid adenocarcinomas, papilla adenocarinomas and carcinoid tumour of lung.

In the present study, at the cut-off point of 0.275 (OD value) on the receiver operating characteristic curve, $52.8 \%$ of squamous cell carcinomas were above this point whereas $97.4 \%$ of adenocarcinomas and $90 \%$ of small cell carcinomas were below this point. None of the benign lung diseases or normal control individuals had S100A7 levels above this threshold (fig 4). Therefore, S100A7 could significantly discriminate squamous cell carcinomas from other cell types of lung cancer, benign lung disease and healthy control groups with $52.8 \%$ sensitivity, $96.3 \%$ specificity and a total accuracy of $98 \%$. Although the
Figure 3 Immunohistochemical analysis of S100A7 protein expression in subtypes of lung cancer tissues, benign lung disease and normal lung tissues. (A-C) Squamous cell carcinoma; (D-E) large cell carcinoma; $(\mathrm{F})$ adenosquamous carcinoma; (G) adenocarcinoma; $(\mathrm{H})$ small cell carcinoma; (I) alveolar cell carcinoma; (J) papilla carcinoma; (K) mucous adenocarcinoma; $(\mathrm{L})$ normal lung tissue; (M-N) pneumonia; $(0)$ normal lung tissue. $(B),(E)$ and $(N)$ are amplified pictures of $(A),(D)$ and $(M)$, respectively.

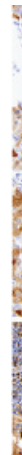

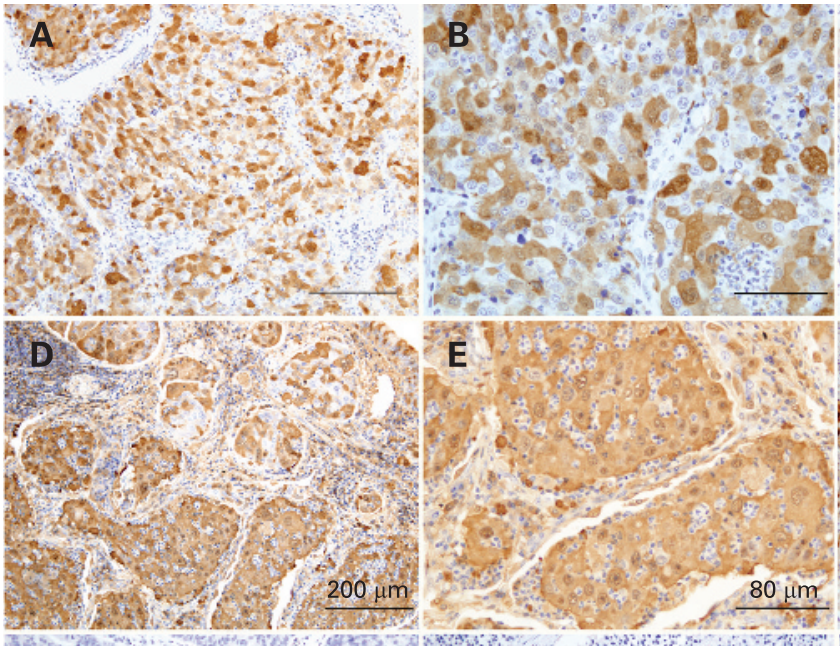

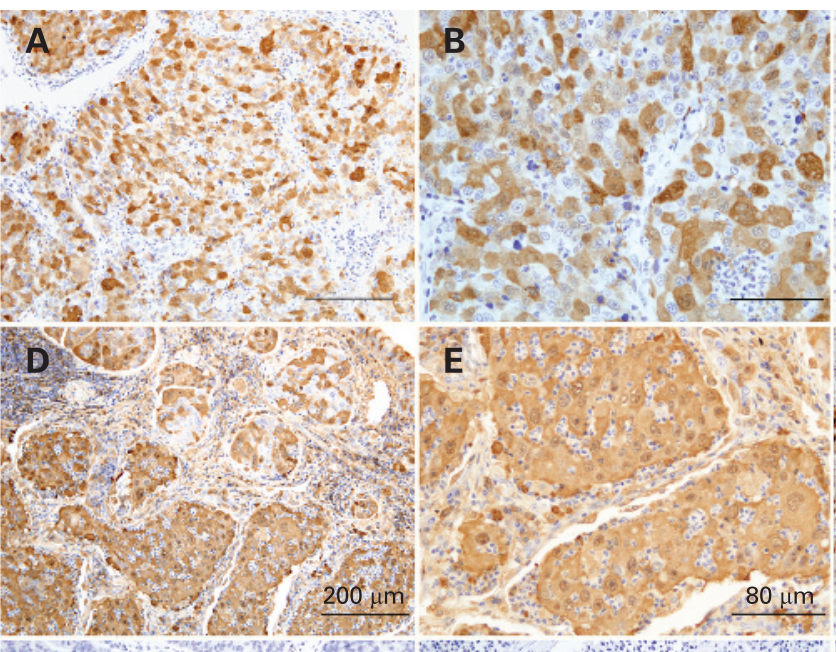
G $\mathrm{G}$

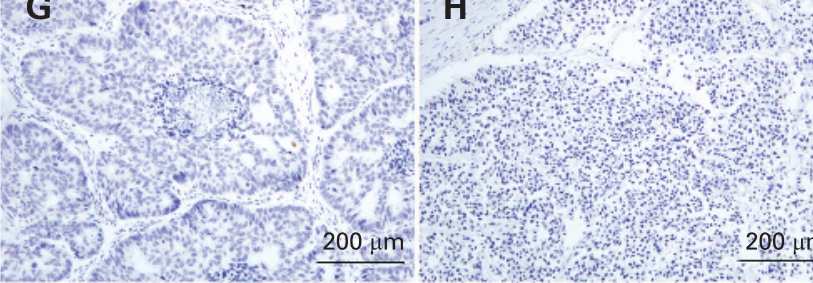
(j)

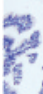
.

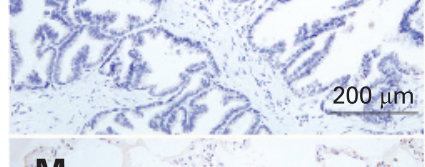

M

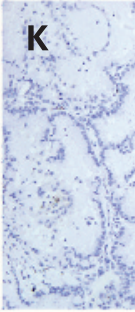

N

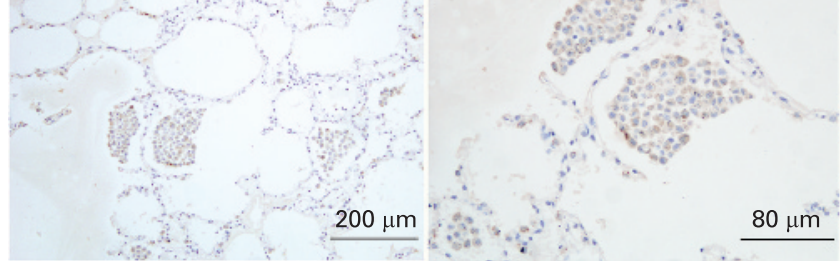

L
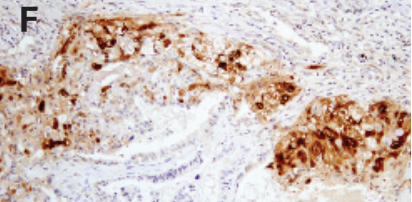

$200 \mu \mathrm{m}$
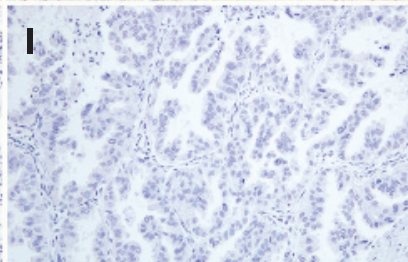

$200 \mu \mathrm{m}$
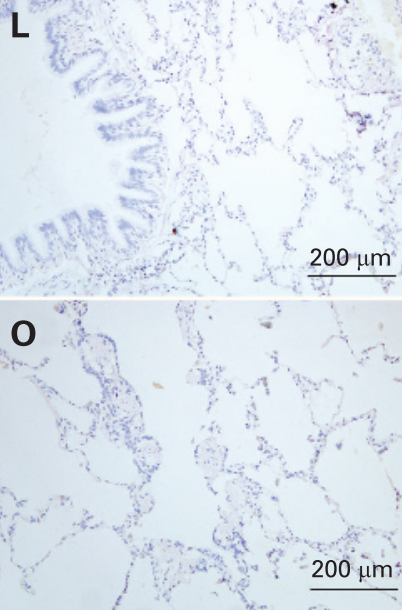
Table 3 Levels of S100A7 in serum samples

\begin{tabular}{lll}
\hline Group & $\begin{array}{l}\text { No serum } \\
\text { samples }\end{array}$ & $\begin{array}{l}\text { S100A7 } \\
\text { (OD values) }\end{array}$ \\
\hline Squamous cell carcinomas & 53 & $0.296(0.119)^{* *}$ \\
I/II & 30 & $0.293(0.103)$ \\
$\quad$ III/IV & 23 & $0.300(0.136)$ \\
Adenocarcinomas & 39 & $0.191(0.052)$ \\
I/II & 19 & $0.190(0.036)$ \\
III/IV & 20 & $0.192(0.065)$ \\
Small cell carcinomas & 20 & $0.183(0.058)$ \\
I/II & 0 & \\
III/IV & 20 & $0.183(0.058)$ \\
Benign lung diseases & 20 & $0.188(0.053)$ \\
Healthy controls & 31 & $0.182(0.046)$ \\
\hline
\end{tabular}

Values are mean (SD).

${ }^{* *} \mathrm{p}<0.001$ compared with the other cell types of lung cancer and control groups.

sensitivity of S100A7 as a diagnostic marker for lung cancer is relatively low, it is a more specific marker of lung squamous cell carcinoma than any other current clinical markers. Taken together, our data strongly suggest that S100A7 can serve as a potential excellent diagnostic marker for detection of lung cancer in serum, even during the early stages of the disease.

\section{DISCUSSION}

S100 proteins are involved in a variety of biological processes and are considered to be expressed in a cell type or tissue specific manner. It is well documented that S100A7 is overexpressed during abnormal skin differentiation, ${ }^{9}$ and is also associated with the early stages or invasion of certain cancers. ${ }^{22-24}$ Overexpression of S100A7 was also found in brain metastases of lung squamous cell carcinomas and primary lung cancer tissues which developed brain metastases 2 6 months after operation. ${ }^{25}$ Based on our previous study and other reported findings, we propose that S100A7 serves not only as an indicator for progression of diseases but may also act as a potential marker for early detection of certain lung cancers.

Unlike other solid tumours, lung cancer is unique in that the different morphological cell types occur at fairly high frequencies. Not uncommonly, difficulty exists in classifying a nonsmall cell lung cancer as adenocarcinoma, squamous cell carcinoma or large cell carcinoma, especially for cancers with poorly differentiated morphologies. ${ }^{26}$ Therefore, identification and characterisation of new lung cancer biomarkers is of great value in cancer diagnosis and therapy, as well as in revealing the mechanisms of carcinogenesis. In the present study, it was important to determine whether S100A7 protein could be differentially expressed in various pathological lung diseases and further validate that S100A7 protein could be detected in sera and may thus act as a potential marker for lung cancer diagnosis. To test this hypothesis, we first demonstrated that both S100A7 mRNA and protein were expressed in squamous cell carcinomas and large cell carcinomas, as well as in adenosquamous cell carcinomas, whereas no S100A7 was found in adenocarcinomas and matched non-cancerous lung tissues. It is therefore reasonable to speculate that S100A7 is likely to be differentially expressed in lung cancer tissues. Our additional studies discovered that immunoreactivity of S100A7 was detected only in squamous cell carcinomas and large cell carcinomas. Interestingly, further observations revealed that S100A7 immunoreactivity in adenosquamous carcinomas occurred only in the squamous cell carcinoma area and weak staining was also detected in some inflammatory cells in benign

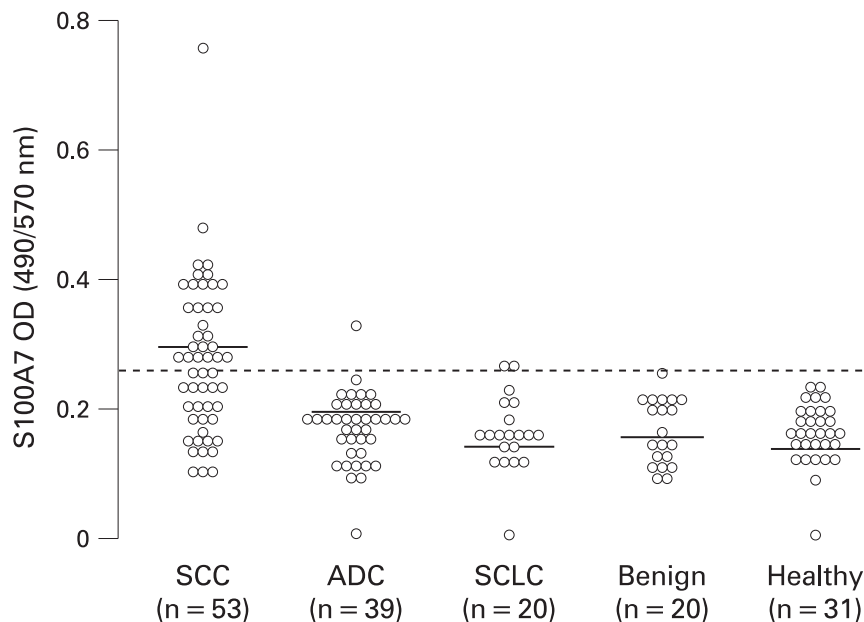

Figure 4 Distribution of the S100A7 OD values in serum samples of patients with lung cancer, benign lung diseases and healthy individuals. ADC, adenocarcinoma; Benign, benign lung diseases; Healthy, healthy individuals; n, number of serum samples; SCC, squamous cell carcinoma; SCLC, small cell lung carcinoma. Broken line is the cut-off value.

lung disease samples, especially the alveolar macrophages from patients with pneumonia. However, no S100A7 expression was observed in other cell types of lung cancer and normal lung tissues. These results strongly support the fact that S100A7 is expressed specifically in subtypes of lung cancer, independent of clinical stage.

It is worth mentioning that a significant increase in S100A7 levels was found in the sera of lung squamous cell carcinoma compared with other cell types of lung cancer, benign lung diseases and healthy individuals. These results provide good supporting evidence for the diagnostic value of the S100A7 protein. The sensitivity and specificity of S100A7 were found to be $52.8 \%$ and $96.3 \%$, respectively, when the cut-off point was set at $0.275(\mathrm{OD})$. However, for two cases of small cell carcinoma, where patients had a slight elevation of serum S100A7, our ELISA method may not have been sufficiently sensitive and accurate compared with other more quantitative techniques. One moderately differentiated adenocarcinoma, stage IV, was found to have moderately increased S100A7 levels undefined in previous studies. In addition, we also found that expression of S100A7 in cancer tissues and its levels in serum were not dependent on the clinical stage of the disease. These data suggest that S100A7 may be an early marker for lung cancer detection. In comparison with recently identified potential markers for lung cancer, such as SAA and TTR, ${ }^{56}$ S100A7 distinguished lung carcinomas and benign lung diseases with a high specificity. Over the past 10 years, other quantative methodologies were also used to detect DNA content, circulating tumour cells, tumour related gene expression, oncogene mutation, microsatellite alterations and promoter methylation in the sera/plasma of patients with lung cancer using real time quantitative PCR. The overall positive detection rates with a single marker were $16 \sim 61 \%$ for patients with lung cancer and $0 \sim 21 \%$ for healthy individuals, with the exception of analysis of free circulating DNA concentration. ${ }^{27-33}$ However, both sensitivity and specificity were markedly increased by detecting circulating cancer cells in peripheral blood with multiple marker genes using either RT-PCR or membrane array based multimarker assay in lung cancer. ${ }^{28}{ }^{34}$ According to these reports and our results, we suggest that levels of S100A7 in sera may serve as 
a helpful candidate for discrimination of lung cancer and nonmalignant lung diseases, while currently used biomarkers fail to provide reliable diagnostic information. An unsatisfactory aspect in the present study was that the majority of frozen tissue samples and paraffin embedded lung cancer tissues were not obtained from the same group of patients. However, it is intriguing that even with this caveat, our data still support the putative diagnostic value of serum S100A7 as a marker for lung squamous cell carcinoma.

The function of S100A7 expressed in lung cancer tissues remains unknown. To interpret our observation, we first hypothesised that S100A7 may be involved in the transformation process of normal bronchial epithelium to squamous cell carcinomas and large cell carcinomas and may thus play an intrinsic role in carcinogenesis. In addition, S100A7 has been reported to alter the activity of activator protein 1 and hypoxia inducible factor 1 transcription factors and their downstream genes in breast cancer cells, consistent with the downstream pathways believed to be regulated by Jab1. ${ }^{35}$ Mutation of three key amino acids (Asp108, Leu130and Asn140) in the Jab1 binding site of S100A7 is known to cause subtle regional structural changes in the protein, in particular in the area around the metal binding sites, which is sufficient to reduce the S100A7 interaction with Jab1 in vivo and in vitro. These mutations have been shown to significantly reduce breast cancer malignant phenotypes in vitro and increase tumorgenicity in vivo in nude mice. ${ }^{17}$ Recently, specific interactions between different S100 proteins were visible by SELDI-MS, and the interaction partners of S100A8/A9 were identified as S100A10 and S100A7, respectively. ${ }^{36}$ In addition to these findings, we predicted that S100A7 protein could be released into the microenvironment of lung cancer tissues, even into the circulating system. The S100A7 protein in the microenvironment may act as a chemotactic factor for inflammatory cells and consequently promote cancer development. S100A7 protein in serum may be developed as a potential biomarker for lung cancer diagnosis. It is known that the interaction between epithelial tumour cells and tumour infiltrating inflammatory cells can be mediated by virtue of factors. It can be produced by malignant tissue and tumour associated macrophages and may thus increase survival, proliferation, migration and invasion of epithelial cancer cells. ${ }^{37}$ Thus it is possible that the secreted S100A7 protein can act as the chemotactic factor and a serum marker for lung cancer.

In summary, we have identified and confirmed that S100A7 is expressed in lung squamous cell carcinomas and large cell carcinomas, but not in adenocarcinomas and small cell lung carcinomas. The S100A7 protein was detected in the serum of patients with lung cancer and its expression was significantly elevated in patients with squamous cell carcinomas. Therefore, the use of S100A7 as a serum biomarker for the detection of lung squamous cell carcinomas may help to facilitate the discrimination of patients with lung cancer from other cell types of lung cancers and patients with benign lung diseases. Elucidation of the biological functions and molecular mechanisms of S100A7 in lung cancer are currently underway in our laboratory.

\begin{abstract}
Acknowledgements: The authors are grateful to Professor Suozhu Sun (Department of Pathology, General Hospital of the Second Artilleryl for evaluating the tissue samples. We thank Professor Jifu Liu and Dr Shanshan Wu (Department of Thoracic Surgery, General Hospital of Beijing Unit, PLA) for providing some tissues and serum samples. We also thank Dr Ligong Duan (General Administration of Sport) for providing the healthy control serum samples.
\end{abstract}

Funding: This work was supported in part by a Special Grant of the Major State Basic Research Program of China (№ 2006CB910100), Project Supported by Natural
Science Foundation of Beijing (№ 7051002) and Project Supported by Science Technology Committee of Beijing (No Y0204002040111).

Competing interests: None.

Ethics approval: The study was approved by the Medical Ethics and Human Clinical Trial Committee at First Hospital of Jilin University and Cancer Hospital, Peking Union Medical College and Chinese Academy of Medical Science, as well as the General Hospital of Beijing Unit, PLA.

\section{REFERENCE}

1. Chen GA, Gharib TG, Wang $\mathrm{H}$, et al. Protein profiles associated with survival in lung adenocarcinoma. Proc Natl Acad Sci U S A 2003;100:13537-42.

2. Granville CA, Dennis PA. An overview of lung cancer genomics and proteomics. Am J Respir Cell Mol Biol 2005;32:169-76.

3. Kulpa J, Wójcik E, Reinfuss $M$, et al. Carcinoembryonic antigen, squamous cell carcinoma antigen, CYFRA21-1, and neuro-specific enolase in squamous cell lung cancer patients. Clin Chem 2002;48:1931-7.

4. Brundage MD, Davies D, Mackillop WJ. Prognostic factors in non-small cell lung cancer: a decade of progress. Chest 2002;122:1037-57.

5. Liu DH, Wang XM, Zhang LJ, et al. Serum amyloid A protein: a potential biomarke correlated with the clinical stage of lung cancer. Biomed Environ Sci 2007;20:33-40.

6. Liu LY, Liu JF, Dai SW, et al. Reduced transthyretin expression in sera of lung cancer Cancer Sci 2007:98:1617-24.

7. Esteller M, Sanchez-Cespedes M, Rosell R, et al. Detection of aberrant promoter hypermethylation of tumor suppressor genes in serum DNA from non-small cell lung cancer patients. Cancer Res 1999;59:67-70.

8. Kulshi JK, Lim CP, Dunn DS, et al. Genomic and phylogenetic analysis of the S100A7 (psoriasin) gene duplications within the region of the $\$ 100$ gene cluster on human chromosome 1q21. J Mol Evol 2003;56:397-406.

9. Madsen P, Rasmussen $\mathrm{HH}$, Leffers $\mathrm{H}$, et al. Molecular cloning, occurrence, and expression of a novel partially secreted protein "psoriasin" that is highly up-regulated in psoriatic skin. J Invest Dermatol 1991;97:701-12.

10. Lee KC, Eckert RL. S100A7 (psoriasin)-mechanism of antibacterial action in wounds. J Invest Dermatol 2007;127:945-57.

11. Semprini S, Capon F, Bovolenta S, et al. Genomic structure, promoter characterization and mutational analysis of the S100A7 gene: exclusion of a candidate for familial psorasis susceptibility. Hum Genet 1999;104:130-4.

12. Alowami S, Qing G, Emberley E, et al. Psoriasin (S100A7) expression is altered during skin tumorigenesis. BMC Dermatol 2003:3:1-7.

13. Celis JE, Rasmussen $\mathrm{HH}$, Vorum $\mathrm{H}$, et al. Bladder squamous cell carcinomas express psoriasin and externalize it to the urine. J Urol 1996;155:2105-12.

14. Al-Haddad S, Zhang Z, Leygue E, et al. Psoriasin (S100A7) expression and invasive breast cancer. Am J Pathol 1999;155:2057-66.

15. Enerbäck C, Porter DA, Seth P, et al. Psoriasin expression in mammary epithelial cells in vitro and in vivo. Cancer Res 2002;62:43-7.

16. Emberley ED, Niu YL, Njue C, et al. Psoriasin (S100A7) expression is associated with poor outcome in estrogen receptor-negative invasive breast cancer. Clin Cancer Res 2003;9:2627-31.

17. Emberley ED, Niu YL, Curtis L, et al. The S100A7-C-Jun activation domain binding protein 1 pathway enhances prosurvival pathways in breast cancer. Cancer Res 2005; 65:5696-702.

18. Porre S, Heinonen S, Mäntyjärvi R, et al. Psoriasin, a calcium-binding protein with chemotactic properties is present in the third trimester amniotic fluid. Mol Hum Reprod 2005;11:87-92.

19. Ostergaard M, Wolf $\mathrm{H}$, Orntoft TF, et al. Psoriasin (S100A7): a putative urinary marker for the follow up patients with bladder squamous cell carcinomas. Electrophoresis 1999:20:349-54.

20. Jinquan T, Vorum H, Larsen CG, et al. Psoriasin: a novel chemotactic protein. J Invest Dermatol 1996;107:5-10.

21. Mountain CF. Revisions in the international system for staging lung cancer. Chest 1997;111:1710-17

22. Emberley ED, Murphy LC, Watson PH. S100A7 and the progression of breast cancer. Breast Cancer Res 2004;6:153-9.

23. Leygue $\mathbf{E}$, Snell L, Hiller T, et al. Differential expression of psoriasin messenger RNA between in situ and invasive human breast carcinoma. Cancer Res 1996;56:4606-9.

24. Krop I, März A, Carlsson $\mathrm{H}$, et al. A putative role for psoriasin in breast tumor progression. Cancer Res 2005;65:11326-34.

25. Zhang $\mathbf{H}$, Wang YP, Chen Y, et al. Identification and validation of S100A7 associated with lung squamous cell carcinoma metastasis to brain. Lung Cancer 2007; 57:37-45

26. Hu R, Wu R, Deng J, et al. A small proline-rich protein, spr1: specific marker for squamous lung carcinoma. Lung Cancer 1998;20:25-30.

27. Sher YP, Shih JY, Yang PC, et al. Prognosis of non-small cell lung cancer patients by detecting circulating cancer cells in the peripheral blood with multiple marker genes. Clin Cancer Res 2005;11:173-9.

28. Sozzi G, Conte D, Leon ME, et al. Quantification of free circulating DNA as a diagnostic marker in lung cancer. J Clin Oncol 2003;21:3902-8.

29. Sueoka $\mathbf{E}$, Sueoka N, Iwanaga K, et al. Detection of plasma hnRNP B1 mRNA, a new cancer biomarker, in lung cancer patients by quantitative real-time polymerase chain reaction. Lung Cancer 2005:48:77-83. 
30. Andriani F, Conte D, Mastranoelo T, et al. Detecting lung cancer in plasma with the use of multiple genetic markers. Int J Cancer 2004;108:91-6.

31. Sozzi G, Conte D, Mariani L, et al. Analysis of circulating tumor DNA in plasma as diagnosis and during follow-up of lung cancer patients. Cancer Res 2001:61:4675-8.

32. Esteller M, Sanchez-Cespedes M, Rosell R, et al. Detecting of aberrant promoter hypermethylation of tumor suppressor genes in serum DNA from non-small cell lung cancer patients. Cancer Res 1999:59:67-70.

33. Bremnes RM, Sirera R, Camps C. Circulating tumour-derived DNA and RNA markers in blood: a tool for early detection, diagnostics, and follow-up? Lung Cancer 2005;49:1-12.
34. Sheu CC, Yu YP, Tsai JR, et al. Development of a membrane array-based multimarker assay for detection of circulating cancer cells in patients with non-small cell lung cancer. Int J Cancer 2006;119:1419-26.

35. Emberley ED, Niu YL, Leygue E, et al. Psoriasin interacts with Jab1 and influences breast cancer progression. Cancer Res 2003;63:1954-61.

36. Lehmann R, Melle C, Eggeling FV, et al. Detection and identification of protein interactions of $\mathbf{S} 100$ proteins by Proteinchip technology. J Proteome Res 2005; 4:1717-21.

37. Shaykhiew R, Bals R. Interaction between epithelial cells and leukocytes in immunity and tissue homeostasis. J Leukoc Biol 2007:82:1-15.

\section{Pulmonary puzzle}

\section{A 75-year-old man with chest discomfort and a cough}

\section{CLINICAL PRESENTATION}

A 75-year-old man was admitted with a non-productive cough and chest discomfort. He had been admitted 2 months previously with left lower lobe (LLL) consolidation for which he received intravenous antibiotics. He had no weight loss or constitutional symptoms. The chest radiograph (fig 1) revealed persistent consolidation and he was urgently referred to the chest clinic for review.

He was an ex-smoker with a 50 packyear history. 12 years previously he was diagnosed with carcinoma of the piriform fossa which was successfully treated with pharyngolaryngectomy, radical radiotherapy and subsequent tracheostomy. Examination revealed evidence of his previous surgery and a tracheostomy. $\mathrm{He}$ had no clubbing or lymphadenopathy and coarse crackles were heard at the left base, as on the previous admission. After treatment with co-trimoxazole for 3 weeks his chest radiograph remained unchanged. A CT scan of the thorax (fig 2) revealed LLL collapse with dense consolidation in the posterior aspect of the right

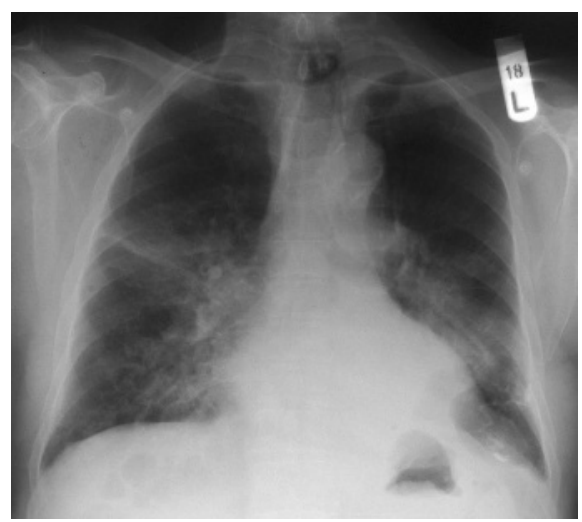

Figure 1 Chest radiograph showing persistent consolidation. middle and lower lobes. The possibility of aspiration was suggested. Bronchoscopy was performed via his tracheostomy which showed a normal right bronchial tree and only a moderate amount of thick white sputum on the left which was easily cleared by washing. Washings were negative for cytology, culture and acidfast bacilli.

CT-guided biopsy (fig 3) showed fibrosis, elastosis and numerous macrophages

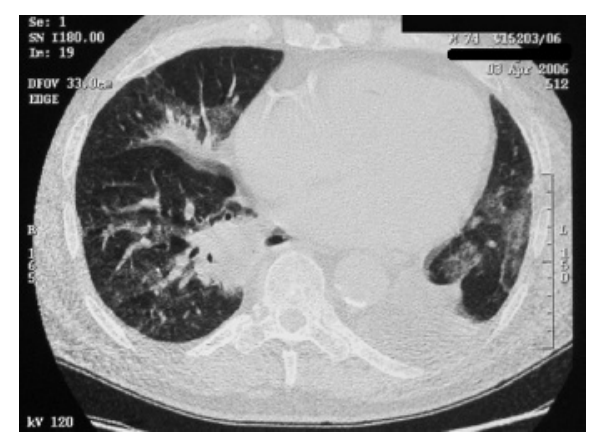

Figure 2 CT scan of the thorax showing left lower lobe collapse with dense consolidation in the posterior aspect of the right middle and lower lobes.

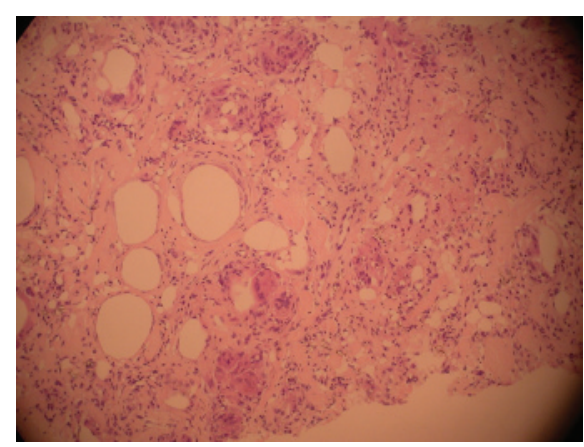

Figure 3 CT-guided biopsy specimen (H\&E stain) showing fibrosis, elastosis, macrophages several multinucleated giant cells surrounding spaces, presumed to contain fat.

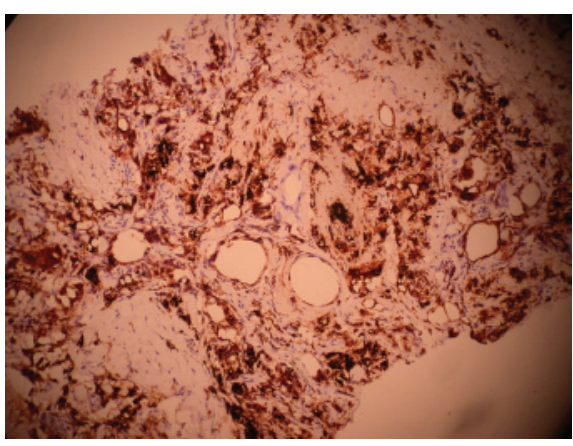

Figure 4 Immunostaining for CD68 which highlights the numerous macrophages.

(CD68 positive, fig 4) with several multinucleated giant cells surrounding spaces, presumed to contain fat. Black carbon pigment was seen in the interstitium and within macrophages. Occasional associated needle-shaped refractile crystals were present and a chronic inflammatory cell infiltrate of lymphocytes. There was no evidence of malignancy.

\section{QUESTION}

Is there anything further you would like to ask in the history and can you suggest a possible diagnosis?

$$
\begin{aligned}
& \text { See page } 376 \text { for answer } \\
& \text { This case was submitted by: }
\end{aligned}
$$

\section{J J Owen, ${ }^{1}$ L Perry, ${ }^{2}$ R Delaney, ${ }^{2}$ M Charig, ${ }^{3}$ M J Smith, ${ }^{4}$ L Hart $^{4}$}

${ }^{1}$ Department of Respiratory Medicine, Queen Alexandra Hospital, Portsmonth Hospitals NHS Trust, UK; ${ }^{2}$ Department of Histopathology, Heatherwood and Wexham Park Hospitals NHS Trust, UK; ${ }^{3}$ Department of Radiology, Heatherwood and Wexham Park Hospitals NHS Trust, UK; ${ }^{4}$ Department of Respiratory Medicine, Heatherwood and Wexham Park Hospitals NHS Trust, UK

Correspondence to: $\mathrm{Dr} J \mathrm{~J}$ Owen, Department of Respiratory Medicine, Trafalgar Building, Queen Alexandra Hospital, Southwick Hill Road, Cosham PO6 3LY, UK; drjowen@ic24.net

\section{Competing interests: None}

Thorax 2008;63:359. doi:10.1136/thx.2007.087668 